Journal of Applied Pharmaceutical Science Vol. 4 (10), pp. 110-116, October, 2014

Available online at http://www.japsonline.com

DOI: $10.7324 / J A P S .2014 .401020$

ISSN 2231-3354 (cc) EY-NC-SA

\title{
Surface activity as basis for pharmaceutical applications of hydrocolloids: A review
}

\author{
Emmanuel O. Olorunsola, Musiliu O. Adedokun \\ Department of Pharmaceutics and Pharmaceutical Technology, Faculty of Pharmacy, University of Uyo, Uyo, Nigeria
}

\author{
ARTICLE INFO \\ Article history: \\ Received on: 31/07/2014 \\ Revised on: 16/08/2014 \\ Accepted on: 03/09/2014 \\ Available online: $30 / 10 / 2014$ \\ Key words: \\ hydrocolloids; \\ pharmaceutical applications; \\ surface activity.
}

\begin{abstract}
Hydrocolloids are polymeric substances with mild to moderate surface activity. They are widely used as excipients in drug delivery systems where they serve various purposes such as suspending, emulsifying, wetting, foaming, solubilizing, stabilizing and bioadhesive agents as well as permeation enhancers. The surface activity and pharmaceutical applications of some hydrocolloids were reviewed in this study. The review showed that most natural hydrocolloids are characterized by higher critical micelle concentrations (CMC) compared to semisynthetic and synthetic ones. Cashew and khaya gums (exudates gums) with hydrophile - lipophile balance (HLB) values in the range of 15 - 18 possess solubilizing property. Dispersions of afzelia and prosopis gums (seed gums) have higher viscosity compared to acacia gum and may produce more stable disperse systems. Semisynthetic and synthetic hydrocolloids like sodium carboxyl methylcellulose and polyvinylpyrrolidone are characterized by low CMC and exhibit very high surface elasticity at concentrations above CMC thus exhibiting high bioadhesive strength. Therefore, surface activity is the basis for most pharmaceutical applications of hydrocolloids and the application of individual hydrocolloid depends on its adsorption power, CMC, HLB value and bioadhesive strength.
\end{abstract}

\section{INTRODUCTION}

Hydrocolloids are extensively utilized in the formulation of many drug delivery systems including solid, liquid and semisolid formulations (Ibezim et al., 2006; Mahmud et al., 2009). They are used due to their low cost, non-toxicity, high biodegradability and high compatibility with drugs (Ofori-Kwakye et al., 2010). They are essential components of many pharmaceutical formulations and serve various purposes in the delivery of drugs.

Drug delivery encompasses the approach, formulation, technology and system involved in transporting drugs into the body. It does not just ensure that drug is presented in a form in which it can be administered but also ensures its release from the dosage form, its absorption and its transportation to the site of action (Ashford, 2007a; Ashford, 2007b).

\footnotetext{
* Corresponding Author

Emmanuel O. Olorunsola, Department of Pharmaceutics and

Pharmaceutical Technology, Faculty of Pharmacy, University of Uyo,

Uyo, Nigeria.Email: olorunsolaeo@yahoo.com
}

Hence, there must be unique characters possessed by hydrocolloids to enable their extensive use in drug delivery. Surface activity is one of the most important of these characters and it is the basis for most of the pharmaceutical applications of these substances (Olorunsola et al., 2014).

The study of interfaces has eventually developed into a separate branch of chemistry, called "Surface Chemistry". This came as a result of the increasing interest in the science and its wide industrial applications such as in textile, food, drink and pharmaceutical industries (Munoz et al., 2007). Hydrocolloids are widely used in these industries (Builders et al., 2009; Ogaji et al., 2012) because of their hydrophilicity, rheological properties and surface activity (Munoz et al., 2007). Therefore, surface activity is a phenomenon that is intimately related to pharmaceutical applications of hydrocolloids.

This study was aimed at reviewing surface science as well as pharmaceutical applications of hydrocolloids and also highlighting the surface properties and surfactant applications of some hydrocolloids. 


\section{SURFACE ACTIVITY}

Surface activity is the ability of a substance to alter the nature of a surface or an interface between two substances. The surface of a pure liquid has a tendency to contract as a result of surface tension forces. Packing of the surface with a surfactant favours expansion of the surface and the surfactant reduces the surface tension of the liquid by an amount equal to the surface pressure (Olorunsola et al., 2014).

Surfactants are characterized by having two distinct regions (hydrophilic and hydrophobic regions) and are termed amphiphiles. They have a tendency to accumulate at the boundary between two phases because of this unique physico-chemical nature. Their adsorption at various interfaces results in changes in the nature of the interfaces making them of great importance in Pharmacy (Attwood, 2007).

Based on the nature of their hydrophilic group, surfactants are generally classified into three, namely: anionic, cationic and non-ionic surfactants. Cationic surfactants are positively charged. Examples of these are gelatin and quaternary ammonium compounds (Adikwu et al., 2005). Anionic surfactants are negatively charged and examples include agar, tragacanth, acacia and sodium lauryl sulphate. Nonionic surfactants are uncharged and are relatively more compatible with drugs. Examples are the polysorbates (Attwood, 2007).

\section{Evaluation of surface activity}

The surface activity of surfactant which is the extent to which it can reduce the surface tension of water is usually evaluated by dissolving it in water and then determining the surface tension of the solution using a tensiometer. Several methods are available for this procedure. These include the plate method, the ring method, drop weight and drop volume method and the capillary rise method. The reduction in the surface tension of water so determined is a measure of the surface activity of the surfactant (Olorunsola et al., 2014).

\section{Plate method}

This is popularly called Wilhelmy plate method. The apparatus consists of thin mica rectangular plate attached to a suitable balance. The plate is immersed into the liquid (whose surface tension is to be determined) and the liquid container is gradually lowered to a point at which the plate lies just at the surface of the liquid and the reading is zeroed. The plate is gradually raised until it just detached from the liquid and the reading on the balance is taken. The surface tension is obtained using the relationship illustrated by equation 1 (Fell, 2007).

$$
\mathrm{F}=2(\mathrm{~L}+\mathrm{B}) \gamma \text {. }
$$

where $\mathrm{F}$ is the detachment force, $\mathrm{L}$ is the length of plate, $\mathrm{B}$ is the breadth of the plate and $\mathrm{y}$ is the surface tension.

\section{Ring method}

The initial apparatus used is called du Nuoy tensiometer. Different forms of this tensiometer are now available. The principle involves measuring the force required to detach a platinum ring from a surface. The ring lying horizontally above the surface is immersed into the liquid. It is gradually raised until it gets to the surface and the reading is zeroed. Thereafter, the ring is further raised until the meniscus of liquid raised by the ring just detached and the force applied is read from the tensiometer (Olorunsola et al., 2014).

As the shape of the liquid supported by the ring is complex and the surface tension force does not act vertically, a correction factor is applied for accurate determination (Adikwu et al., 2005). The surface tension is calculated using equation 2 .

$$
\mathrm{\gamma}=\frac{F}{2 \pi R} \cdot \emptyset
$$

where $\mathrm{y}$ is the surface tension, $\mathrm{F}$ is the detachment force, $\mathrm{R}$ is radius of the ring and $\varnothing$ is the correction factor of the instrument.

\section{Drop weight and drop volume method}

Here, the volume and weight of a drop detached from a tip of known radius is determined. The tip must be completely wetted by the liquid, the drop must not climb outside the tube and should be formed slowly to get accurate result. According to Fell (2007), the surface tension is calculated using equation 3 .

$$
\mathrm{\gamma}=\frac{\emptyset m g}{2 \pi r}=\frac{\emptyset V \rho g}{2 \pi r}
$$

where $\mathrm{v}$ is surface tension, $\mathrm{m}$ is the mass of the drop, $\mathrm{v}$ is the volume of the drop, $\rho$ is the density of the liquid, $r$ is the radius of the tip, $g$ is acceleration due to gravity and $\varnothing$ is the correction factor.

\section{Capillary rise method}

This is seldom used in pharmaceutical research. A capillary tube is placed in the liquid whose surface tension is to be measured. The liquid is allowed to rise in the tube to a certain length and the surface tension is calculated using equation 4 (Fell, 2007).

$$
\mathrm{\gamma}=\frac{r_{t} h\left(\rho_{L}-\rho_{V}\right) g}{2}
$$

where $r_{t}$ is the radius of the capillary tube, $h$ is the height of liquid in the capillary tube, $\rho_{L}-\rho_{V}$ is the density different between the liquid and its vapour and $g$ is acceleration due to gravity.

\section{CRITICAL MICELLE CONCENTRATION (CMC)}

Critical micelle concentration (CMC) is the concentration above which aggregates of colloidal dimension, called micelles, are formed. To determine the CMC of a surfactant, a graph of surface tension of solutions of increasing concentration of the surfactant is plotted against natural logarithm of the concentrations. Generally, surface tension decreases with increase in surfactant concentration until a point where further increase in concentration does not affect the surface tension. This point is termed critical micelle concentration. Accumulation in surface layer and adsorption at interface are favoured below CMC while 
micelle formation and solubilization are favoured above CMC (Attwood, 2007). When surface active agents are present below their CMC, they concentrate at the water surface with the hydrophobic region orientated away from the aqueous phase causing an expansion of the surface layer. This leads to reduction in the surface tension of the liquid (Dominguez et al., 1997). When they are present above the CMC, they form micelles. The hydrophobic chains of the surfactant form the core of the micelles and are shielded from the aqueous environment by the hydrophilic chains (Dominguez et al., 1997). Formation of micelles is responsible for solubilization of water-insoluble substances. This is the principle behind the use of surface active agents as solubilizing agents.

\section{HYDROPHILE-LIPOPHILE BALANCE (HLB)}

Hydrophile-lipophile balance (HLB) is the relative hydrophilicity or lipophilicity of a surfactant. It is characteristic of the relative polarity of the surfactant and ranges from 0 to 20 . The lower the HLB value, the more hydrophobic is the surfactant and the higher the HLB value, the more hydrophilic is the substance. HLB values have been used to classify surfactants as water-in-oil emulsifiers, wetting agents, oil-in-water emulsifiers, foaming agents and solubilizing agent in order of increasing HLB values (Davies, 1957):

\begin{tabular}{ll}
- & Water-in-oil emulsifiers (HLB values of 4-6) \\
- & Wetting agents (HLB value of 7-9) \\
- & Oil-in-water emulsifiers (HLB values of 8-18) \\
- & Foaming agents (HLB values of 13-15) \\
\hline & Solubilizing agents (HLB values of 15-18).
\end{tabular}

\section{USES OF SURFACTANTS}

The different uses of surfactants depend on their ability to get adsorbed at surfaces, their ability to reduce interfacial tension and their HLB values. They are thus used as:

\section{Emulsifiers}

Water and oil are immiscible liquids. However, emulsification of the two liquids occurs in the presence of an emulsifier. Emulsifiers work by reducing interfacial tension between oil and water molecules. As earlier mentioned, those with low HLB values form water-in-oil emulsions while those with higher HLB values form oil-in-water emulsions. The type of emulsion produced also depends on the quantities of the two liquids available. When the quantity of oil is higher, there is greater tendency for the formation of water-in-oil emulsion. Conversely, there is greater tendency for the formation of oil-inwater emulsion when the quantity of water is higher (Attwood, 2007). While simple emulsions exist as water-in-oil or oil-inwater, multiple emulsions are more complicated and can be waterin-oil-in-water $(\mathrm{w} / \mathrm{o} / \mathrm{w})$ or oil-in-water-in-oil $(\mathrm{o} / \mathrm{w} / \mathrm{o})$ emulsions. Two emulgents are required for the formulation of multiple emulsions. For instance, to prepare w/o/w emulsion, a w/o emulgent and an o/w emulgent are required. The w/o emulgent, for example, sorbitan mono-oleate, is first mixed thoroughly with the oily phase (such as liquid paraffin) in a mixer. This is followed by the slow addition of the aqueous phase (containing the drug) to the oil/emulgent mixture with vigorous mixing to form a w/o emulsion. The w/o emulsion is then dispersed in an aqueous solution of an o/w emulgent such as tween (polysorbate) 80 to obtain the desired w/o/w emulsion.

Multiple emulsions are mainly useful in parenteral therapy to effect drug localization and prolongation of drug action. The drug in the innermost phase diffuses slowly through the other phases to provide sustained release. They are thus used in delayed action drug delivery (Attwood, 2007).

\section{Wetting agents}

These are water miscible solvents. They act by reducing liquid - air interfacial tension to facilitate displacement of air and enable liquid to surround or penetrate into the pores of hydrophobic powdered particles. Non-ionic surfactants with HLB values from 7 - 9 are used as wetting agents (Davies, 1957).

Hydrophilic materials are easily wetted by water while hydrophobic materials are not. However, non-polar liquids wet hydrophobic materials easily. The extent of wetting by water is dependent on the hydrophilicity of the material (Gohel et al., 2005). If the material is more hydrophilic, it will have less difficulty in being wetted by water. Inability of particles of a drug to be wetted reflects high interfacial tension between the drug material and the vehicle being used. Sulphamethoxazole and trimethoprim are examples of powders with poor wettability.

\section{Foaming agents}

Surfactants with HLB values of 13-15 are used as foaming agents. Foam is a disperse system of a gas in a liquid which is present as thin films of colloidal order between the gas bubbles. The film is made up of two monolayers of adsorbed surfactant molecules separated by an aqueous core. Therefore, foaming agents act by causing double-layer repulsion. Foams find application in pharmacy as spray preparations of medicaments for rectal, vaginal and topical administration. Concentration of surfactant used should be less than $0.5 \%$ (or CMC of the surfactant) to avert solubilization of the medicament (Mahmud, 2009).

\section{Solubilizing agents}

Surfactant with HLB values close to 20 are used as solubilizing agents. These are highly polar surfactants used at concentrations above CMC. At concentrations above CMC, the hydrophobic chains of the surfactants form the core of the micelles and are shielded from the aqueous environment by the hydrophilic groups. This brings about solubilization of the water-insoluble compounds. Solubilizing agents are used for the formulation of poorly soluble medicaments (Olorunsola et al., 2014). 


\section{Suspending agents}

Suspending agents act by adsorption on the waterinsoluble particles keeping them dispersed within the dispersion medium (Olorunsola et al., 2014). They are added to suspensions to increase viscosity and density of the dispersion medium and to reduce the settling of dispersed particles.

\section{Stabilizers}

Stabilizers are added to suspensions and emulsions to enhance stability. For suspensions, the rate of separation of the dispersed phase and the dispersing medium is reduced by incorporation of surfactants. For emulsions, phase separation (creaming) and cracking are reduced by incorporation $\boldsymbol{a} \phi^{\mathrm{f}}$ surfactants (Mdhlovu, 2005).

\section{Bioadhesive agents}

The surface energy of surfactants or adhesive polymers used in bioadhesion improves the intimacy between the drug and the biological surface promoting drug delivery (Adikwu et al., 2005). The penetration of the adhesive polymer or surfactant into mucus network and the formation of secondary chemical bonds are influenced by several factors which include the ionic charge of the polymer and the strength of hydrogen bonding between the polymers (Genarro, 1995). Bioadhesion is a state in which two materials at least one of which is of a biological nature are held together for an extended period of time by interfacial forces (Adikwu et al., 2005).

For drug delivery purposes, bioadhesion implies attachment of drug carrier system to a biological location thus increasing drug absorption and bioavailability. Bioadhesive dosage forms are targeted at particular sites such as nasal, buccal, gastrointestinal tract, cervical, vaginal and dermal regions to reduce toxic effects and increase therapeutic effects of drugs (Attama et al., 2003).

\section{Permeation enhancers}

Surface-active agents reversibly interfere with biological membranes increasing their permeability. Therefore, they are incorporated as permeation enhancers in pharmaceutical formulations (solids, liquids and semi-solids) to promote drug absorption. Examples of such surfactants are sodium taurocholate and sodium thioglycollate (Sharma et al., 2013).

\section{HYDROCOLLOIDS}

Hydrocolloids (also called gums) are colloidal substances that are generally non-crystalline in nature. They are watersoluble or water-dispersible hydrophilic compounds which are insoluble in alcohol and organic solvents. They are high molec $\boldsymbol{b l}$ ) hr weight polysaccharides which are formed from sugars and uronic acid units. They may also contain potassium, sodium, calcium and magnesium salts (Ofori-Kwakye et al., 2010).

\section{Classification of gums}

Gums can be classified into natural gums, semi-synthetic gums and synthetic gums. This classification is based on their sources and mode of production (Ofori-Kwakye et al., 2010).

\section{Natural gums}

These are gums that are obtained from living organisms (plants, animals and micro-organisms) and are formed by natural processes. The major limitation to their use as pharmaceutical excipients is their high susceptibility to microbial attack (Billany, 2007).

\section{Plant gums}

A wide variety of plants are known for gum production. Plant gums are produced either as exudates, seed gums, seaweed gums or as pectin.

Exudates are produced by a process called "gummosis". Gummosis is the formation of patches of gluey substances on the surface of certain plant barks. It occurs as a result of wound inflicted on the plant by external stimuli, adverse weather conditions, microbial attack, insect attack or other mechanical damages. Subsequently, sap exudes from the wound a sticky substance which on exposure to air, solidify to form amorphous translucent solids called gum (Mahmud, 2009).

Seed gums are obtained majorly from seeds of some plants in the family Fabaceae (Builders et al., 2009; Ezike et al., 2010). Prosopis africana and Afzelia africana are examples of plants with gum-yielding seeds. Seed gums are hemicellulosic in nature (Builders et al., 2009).

Seaweed gums are gums that are extracted from seaweed. Examples are alginates and agar. Alginates are natural polysaccharide polymers isolated from the brown seaweed (Phaeophyceae). Alginic acid can be converted into its salts, of which sodium alginate is the commonest one in use. They are linear polymers consisting of D-mannuronic acid and L-glucuronic acid residues arranged in blocks in the polymer chain. They offer various applications in drug delivery (Ogaji et al., 2012). Agar is extracted from red-purple aquatic algae of the class Rhodophyceae. The species include Gelidium cartilagineum and Gracilaria confervoides which grow abundantly in the waters along the coast of Japan, New Zealand, South Africa, Southern California, Mexico, Chile, Morocco, and Portugal (Ogaji et al., 2012).

Pectins are gums that are extracted from citrus fruits. They are predominantly linear polymers of mainly (1-4)-linked Dgalacturonic acid residues interrupted by 1,2- linked L-rhamnose residues. Each molecule contains a few hundreds to about one thousand building blocks (Ogaji et al., 2012).

\section{Animal gums}

Gums derived from animal skin and bones are called gelatin. Unlike plant gums that are mostly anionic or non-ionic, gelatin has a net positive charge (Adikwu et al., 2005). 


\section{Gums of microbial origin}

Microbial gums are produced by fermentation process. Xanthan gum, a complex microbial exopolysaccharide is produced from glucose fermentation by Xanthomonas campestrispv. The gum consists of D-glucosyl, D-mannosyl, and D-glucuronyl acid residues in a molar ratio of 2:2:1. It also contains o-acetyl and pyruvyl residues in variable proportions. Xanthan gum is an acidic polysaccharide gum of penta-saccharide subunits (Ogaji et al., 2012).

Gellan gum is an anionic microbial polysaccharide, secreted by Sphingomonas elodea, consisting of repeating tetrasaccharide units of glucose, glucuronic acid and rhamnose residues in a 2:1:1 ratio. Pullulan is an extracellular homopolysaccharide of glucose produced by many species of the fungus Aureobasidium, specifically A. pullulans (Ogaji et al., 2012).

\section{Semi-synthetic or modified gums}

These are produced by chemical modification of natural gums. Cellulose derivatives can be made by etherification, esterification, cross-linking or graft copolymerization. Etherification yields derivatives such as hydroxyl-propylmethylcellulose and carboxyl-methylcellulose, while esterification results in derivatives which include cellulose acetate and cellulose acetate phthalate. Cashew gum has been modified by carboxyl methylation using monochloroacetic acid as etherifying agent (Maciel et al., 2005).

\section{Synthetic gums}

These are chemically produced gums. Polyvinyl pyrrolidone is a typical example. Synthetic gums can be used at low concentrations and are less susceptible to microbial attack. These are the major advantages over natural hydrocolloids (Billany, 2007).

\section{SURFACTANT BEHAVIOURS OF SOME HYDROCOLLOIDS}

\section{Acacia gum}

This is a plant exudates gum. The critical micelle concentration of Acacia gum B.P. is $0.25 \% \mathrm{w} / \mathrm{v}$ and the gum is capable of lowering the surface tension of water from 72.0 to 47.9 $\mathrm{mN} / \mathrm{m}$ (Olorunsola et al., 2014). Investigation of surface properties of Acacia tortuosa gum carried out by Munoz et al. (2007) showed the critical micelle concentration of the hydrocolloid as being 0.5 $\% \mathrm{w} / \mathrm{v}$. The work also showed that the surface tension of water was reduced from 72.5 to $42.6 \mathrm{mN} / \mathrm{m}$ at this surfactant concentration.

Researchers have focused on comparative studies of suspending and emulsifying abilities of different Acacia species, for example, comparative evaluation of suspending properties of gum obtained from two species of Acacia, namely, A. senegal and A. sieberiana was carried out by Mahmud (2009). The two gums compared favourably in terms of producing suspensions with low sedimentation rate at high concentrations. This is an example of surfactant application of the gum.

\section{Cashew gum}

Cashew gum is obtained from exudates of Anacardium occidentale L. Evaluation of surface activity of some hydrophilic polymers using Searl's tensiometer by Olorunsola et al. (2014) showed $0.5 \% \mathrm{w} / \mathrm{v}$ as being the CMC of cashew gum and that the surface tension of the gum dispersion at this concentration was $55.0 \mathrm{mN} / \mathrm{m}$. This shows that it is less surface active compared to Acacia gum at CMC. The work also showed that the gum has HLB value of 16.09. This is within the range of HLB values of oil-inwater emulsifiers. It also falls within the range of HLB values of solubilizing agents. Therefore, cashew gum might possess oil-inwater emulsifying and solubilizing properties. Cashew gum was evaluated as a gelling agent in aceclofenac topical gel formulation. The gel containing $5 \%$ w $/$ cashew gum was found to be suitable for topical application (Kumar et al., 2009).

\begin{abstract}
Albizia gum
Femi-Oyewo and co-workers (2004) investigated the suspending ability of exudates of Albizia zygia Family Mimosoideae comparatively with those of compound tragacanth, acacia and gelatin at concentration range of $0.5-4.0 \% \mathrm{w} / \mathrm{v}$ in sulphadimidine suspension. Albizia gum appeared to exhibit the best suspendability of all the polymers. In fact, $2.5 \% \mathrm{w} / \mathrm{v}$ of this gum produced suspension of optimal properties which compared favourably with the suspension containing $4.0 \% \mathrm{w} / \mathrm{v}$ compound tragacanth, a traditional suspending agent. Mucilage of Albizia gum was thus recommended for use as stabilizer and thickener of choice when high viscosity is desired especially in cosmetic, pharmaceutical and food industries (Femi-Oyewo et al., 2004).
\end{abstract}

\section{Khaya gum}

Khaya gum is obtained from exudates of Khaya senegalensis. Concentration of $0.75 \% \mathrm{w} / \mathrm{v}$ was shown to be the CMC of khaya gum and the surface tension of the gum dispersion at this concentration was found to be $55.0 \mathrm{mN} / \mathrm{m}$ (Olorunsola et al., 2014). Even though cashew and khaya gums have different CMCs, their dispersions at CMC have the same surface tension. The work also showed that khaya gum has HLB value of 15.91. This value is within the range of HLB values of oil-in-water emulsifiers and also within the range of HLB values of solubilizing agents. Cashew gum and khaya gum have these characteristics in common. The two gums are chemically-related in that they are galactan polymers (Lima et al., 2002).

Khaya gum was evaluated for suspending property by Mahmud (2009). Co-trimoxazole suspensions formulated with the gum had the highest sedimentation rate compared to those of Acacia senegal and Acacia sieberiana gums at $2 \% \mathrm{w} / \mathrm{w}$ gum concentration. Suspension containing $5 \% \mathrm{w} / \mathrm{w}$ khaya gum was also found to be more stable compared to that containing $2 \%$ w $/$ gum concentration. 


\section{Afzelia gum}

Afzelia gum obtained from seeds of Afzelia africana has a high swelling capacity and can be used as a hydrogel (Builders $e t$ al., 2009). Dispersion containing $2 \% \mathrm{w} / \mathrm{v}$ of the gum has higher viscosity compared to the same concentration of acacia gum (Ibezim et al., 2006). Pharmaceutical suspensions and emulsions formulated with the gum will be more viscous and less pourable compared to those formulated with the same concentration of acacia gum.

\section{Prosopis gum}

Dispersion containing $2 \% \mathrm{w} / \mathrm{v}$ prosopis gum obtained from seeds of Prosopis africana has higher viscosity compared to the dispersion containing the same concentration of acacia gum. The bioadhesive value of the gum is commensurate with those of Carbopol 974-P and sodium carboxyl methylcellulose (Adikwu et al., 2003). The gum had also been investigated for bioadhesive delivery of theophylline. The results showed that prosopis gum is highly bioadhesive compared to sodium carboxyl methylcellulose and that it could be used to deliver theophylline in a bioadhesive dosage form (Attama et al., 2000).

\section{Okra gel}

Okra gel obtained from fruits of Abelmoscus esculentus, though a natural hydrocolloid has properties similar to sodium carboxyl methylcellulose (SCMC) and hydroxylpropyl methylcellulose (HPMC). Similar concentration $(2 \% \mathrm{w} / \mathrm{v})$ of the three polymers have comparable viscosity. The bioadhesive strength of high concentration of the three polymers (above CMC) is in the order SCMC > okra gel > HPMC (Sharma et al., 2013).

\section{Snail mucin}

Snail mucin, an animal hydrocolloid obtained from Archachatina marginata family Arionidae possesses surface activity as determined using Du Nuoy tensiometer by Adikwu et al. (2005). Dispersions of concentrations above CMC and ranging from 4 to $20 \% \mathrm{w} / \mathrm{v}$ were prepared. Each dispersion was spread over a section of isolated thoroughly washed pig intestine which was later covered with the plate of the tensiometer allowing for contact time of 7 minutes. The force required to detach the plate from the surface of the intestine was determined. The force applied to cause detachment increased with increase in the concentration of mucin (Adikwu et al., 2005) showing that mucin is a good bioadhesive at concentrations above CMC.

\section{Sodium carboxyl methylcellulose}

Sodium carboxyl methylcellulose is sodium salt of a semi-synthetic gum. It has a lower CMC $(0.1 \% \mathrm{w} / \mathrm{v})$ compared to natural gums. This can be attributed to the presence of ions in the molecule. Presence of ions generally decreases critical micelle concentration of surfactant and increases micellar size (Dominguez et al., 1997). This is sequel to the increase in the force of repulsion between the similar charged groups in the micelle; promoting micelle growth and reducing the energy required for their formation (Attwood, 2007).

\section{Polyvinylpyrrolidone}

This is a synthetic gum. The surface activity of this polymer was investigated by Noskor et al. (2002) using Wilhemy plate method. The surface elasticity was found to decrease with increase in the polymer concentration up to $0.1 \% \mathrm{w} / \mathrm{v}$ after which an abrupt increase in elasticity was observed. Therefore, the CMC of the polymer is $0.1 \% \mathrm{w} / \mathrm{v}$.

\section{CONCLUSION}

Hydrocolloids are moderate surface active substances that are extensively used as pharmaceutical excipients. Different hydrocolloids have different applications in different pharmaceutical dosage forms depending on their adsorption power, critical micelle concentration, HLB value and bioadhesive strength. Therefore, surface activity is the basis for most pharmaceutical applications of hydrocolloids and the application of individual hydrocolloid depends on its own surface parameters.

\section{REFERENCES}

Adikwu MU, Yoshikwa Y, Kanji, T. Bioadhesive delivery of metformin using prosopis gum with antidiabetic potential. Biol Pharm Bull, 2003; 26 (5): 662 - 666.

Adikwu MU, Nnamani PO, Attama AA. Evaluation of snail mucin as bioadhesive agent for the delivery of chlorpropamide. BioResearch, 2005; 3 (2): 75 - 85.

Ashford M. 2007a. Gastrointestinal tract - Physiology and drug absorption. In: Aulton ME, ed. The Design and Manufacture of Medicine. Philadelphia: Churchill Livingstone, Elsevier 270 - 285.

Ashford M. 2007b. Bioavailability - physicochemical and dosage form factors. In: Aulton ME, ed. The Design and Manufacture of Medicine. Philadelphia: Churchill Livingstone, Elsevier 286 - 323.

Attama AA, Adikwu MU, Okoli N. Studies in bioadhesive granules 1: Granules formulated with Prosopis africana gum. Chem Pharm Bull, 2000; 48 (5): 734 - 737.

Attama AA, Adikwu MU, Nnamani PO. Delivery of diclofenac sodium via non-disintegrating bioadhesive tablets of paraffin wax. S.T.P. Pharma Sci, 2003; 13 (2): 147-150.

Attwood D. 2007. Disperse systems. In: Aulton ME, ed. The Design and Manufacture of Medicine. Philadelphia: Churchill Livingstone, Elsevier 70-98.

Billany MR. 2007. Suspensions and emulsions. In: Aulton ME, ed. The Design and Manufacture of Medicine. Philadelphia: Churchill Livingstone, Elsevier 383 - 405.

Builders PF, Chukwu C. Obidike I, Builders MI, Attama AA, Adikwu MU. A novel xyloglucan gum from seeds of Afzelia africana Se. Pers.: Some functional and physicochemical properties. Int J Green Pharm, 2009; 3 (2): 112 - 118.

Davies JT. 1957. A quantitative kinetic theory of emulsion type 1: Physical chemistry of the emulsifying agent. Proceedings of Second International Congress on Surface Activity. London: Butterworth.

Dominguez A, Fernandez A, Gonzalez N, Iglesias E, Montenegro L. Determination of critical micelle concentration of some surfactants by three techniques. J Chem Edu, 1997; 74(10): 1227-1231.

Ezike AC, Akah PA, Okoli CO, Udegbunam S, Okumni N, Okeke C, Iloan O. Medicinal plants used in wound dressing : A case study of Prosopis africana (Fabaceae) stem bark. Ind J Pharm Sci, 2010; 72 (3): 334 - 339. 
Fell JT. 2007. Surface and interfacial phenomena. In: Aulton ME, ed. The Design and Manufacture of Medicine. Philadelphia: Churchill Livingstone, Elsevier 59-69.

Femi-Oyewo MN, Adedokun MO, Olusoga TO Evaluation of the suspending properties of Albizia zygia gum on sulphadimidine suspension, Trop J Pharm Res, 2004; 3 (1): 279 - 284

Florence AT, Attwood D. 2007. Physicochemical Principles of Pharmacy, 4th edition. London: Pharmaceutical Press 177-228.

Genarro AR. 1995. Remington - The Science and Practice of Pharmacy. 19th ed. Volume II, Mack Publishing Company, Exton, Penysylvania, 8992.

Gohel M, Patrikh R, Patel C, Joshi H. 2005. Pharmaceutical Suspensions - A Review. www.pharmainfo.net/freebooks.

Ibezim EC, Khanna M, Singh S, Uzuebenam CE. Afzelia africana seed gum: potential binder for tablet formulations. J Phytomed Thera, 2006; 11: 38 - 48.

Kumar R, Patil MB, Patil SR, Paschapur MS. Evaluation of Anacardium occidentale gum as gelling agent in aceclofenae gel. Int $J$ Pharm Tech Res, 2009; 1(3) : 695 - 704.

Lima RDN, Lima JR, Desalis CR, Moreira RA. Cashew tree (Anacardium occidentale L.) exudates gum: A novel bioligand tool. Biotech Appl Biochem, 2002; 35 : 45 - 53.

Maciel JS, Silva DA, Paulla HCB, dePaula RCM. Chistosan/carboxyl methyl cashew gum polyelectrolyte complex: Synthesis and thermal stability. Eur Polym J, 2005; 41 (11): 2726 - 2733.

Mahmud HS. 2009. Evaluation of Acacia sieberiana and Khaya senegalensis gum in formulation of pharmaceutical suspensions. Unpublished M.Sc. Thesis (Ahmadu Bello University, Zaria).

Mahmud HS, Oyi AR, Allagh TS. Evaluation of the suspending property of Khaya senegalensis gum in formulation of paracetamol suspension. Nig J Pharm Sci, 2009; 8 (1): 128 - 134.
Mdhlovu J. 2005. Optimization of Water-in-oil Micro-emulsion Stabilized by Nonylphenol Ethoxylated Phosphate Ester. PhD Thesis. University of Pretoria, South Africia.

Munoz J, Rincon F, Alfaro MC, Zapata I, Fuente J, Beltran O, dePinto GL. Rheological properties and surface tension of Acacia tortuosa. Carbohydrate Polym, 2007; 70: 198 - 205.

Noskor BA, Akentier AV, Miller R. Dynamic surface properties of polyvinylpyrrolidone solution. J Colloid Interface Sci, 2002; 255(2): 417 - 424.

Ofori-Kwakye K, Asantewaa Y, Kipo SL. Physicochemical and binding properties of cashew tree gum in metronidazole tablet formulation. Int J Pharm Pharm Sci, 2010; 2 (4): 105 - 109.

Ogaji IS, Nep EI, Audu-Peters JD. Advances in natural polymers as excipients. Pharm Analytica Acta, 2012; vol. 3 art. 146.

Olorunsola EO, Bhatia PG, Tytler BA, Adikwu MU. Surface activity and hydrophile-lipophile balance of hydrophilic polymers from exudates of cashew and khaya plants. Int J Pharm Biol Res, 2014; 5(5): $443-448$.

Sharma N, Kulkarni GT, Sharma A. Development of novel Abelmoschus esculentus (Okra) gel for nasal delivery of rizatriptan benzoate. Trop J Pharm Res, 2013; 12 (2): 149 - 153.

\section{How to cite this article:}

Olorunsola EO, Adedokun MO. Surface activity as basis for pharmaceutical applications of hydrocolloids: A review. J App Pharm Sci, 2014; 4 (10): 110-116. 